\title{
Modifications on Impression Procedure and Occlusal Scheme for Complex Oral Conditions in Complete Edentulous Patient
}

${ }^{1}$ Chihargo, ${ }^{2}$ Ismet D Nasution, ${ }^{3}$ Ricca Chairunnisa

\section{ABSTRACT}

Background: Patient's perspective is analyzed on the treatment options when invasive treatments, such as implant, bone grafting, or vestibuloplasty become contraindication, and a noninvasive approach, such as conventional complete dentures became treatment of choice that could be done.

Aim: This clinical report describes a modification of impression procedure and modification of occlusal scheme in fabricating complete dentures to manage flabby ridge, flat ridge, and prognathic jaw relation with posterior cross-bite at once in complete edentulous patient.

Case report: A 66-year-old female patient was wearing single upper complete denture since her lower complete denture was broken a year ago. On clinical examination, there were flabby ridge on anterior and flat ridge on posterior residual ridges of maxilla. Moderate resorption was found on posterior mandibular residual ridges. Prognathic jaw relation with posterior cross-bite was discovered from tentative vertical dimension (VD) procedure.

Conclusion: Modified impression procedure, which was used in this case for flabby and flat ridge on maxilla using clear processed acrylic resin tray with controlled minimally displacive impression technique, provides mucofunctional impression on posterior and mucostatic impression on anterior. Anterior artificial teeth were arranged to neutrognathic jaw relation without vertical overlap to avoid pressure on the flabby ridge. Modified lingualized occlusion was used to overcome flat ridge with posterior cross-bite.

Clinical significance: Modified impression procedure used in this case was simple and can record abnormal residual ridges in its compressibility. The modified lingualized occlusion can help maintain the occlusal forces right on the crest of residual ridges without interfering the neutral zone. Hence, fabrication of a stable and satisfying denture can be achieved.

Keywords: Flabby ridge, Flat ridge, Progenic jaw.

How to cite this article: Chihargo, Nasution ID, Chairunnisa R. Modifications on Impression Procedure and Occlusal Scheme for Complex Oral Conditions in Complete Edentulous Patient. World J Dent 2018;9(2):126-131.

Source of support: Nil

Conflict of interest: None

\footnotetext{
${ }^{1-3}$ Department of Prosthodontics, Faculty of Dentistry, Universitas Sumatera Utara, Medan, Indonesia

Corresponding Author: Chihargo, Jl. Alumni No. 2 Kampus USU P.O. Box 20155, Medan, Indonesia, Phone: +6281912541758 e-mail: chihargo89@gmail.com
}

\section{BACKGROUND}

Retention, stability, and support are considered the most important factors for the clinical success of complete dentures. Excessive movable tissue or flabby tissue is easily displaced, which will adversely affect the retention, stability, and support of complete dentures. ${ }^{1}$ The degree of muscular activity and the region to which the denture can be extended without displacement are important aspects of any impression technique. For individuals with a severe bone resorption or flat ridge, it is difficult to obtain good retention and stability of the complete denture due to the presence of muscular insertions near the ridge crest or border, which might cause muscularinduced displacement of the denture. ${ }^{2}$ Therefore, modifications on impression procedure, especially on custom tray and impression techniques which will be used for final impression, are needed to prevent excessive pressure and distortion on abnormal tissues.

The speed and direction of alveolar bone resorption are not similar in maxilla and mandible. Faster and more dramatic changes take place in the mandible. In maxilla, the changes occur evenly around the dental arch, but more on buccal and labial side than on the palatal side. In mandible, resorption precedes more in labiolingual and vertical directions. Thus, it could create an appearance of prognathic jaw relation also in cross-bite situation on posterior ridges in fully edentulous patient. ${ }^{3}$

Lingualized occlusion can be defined as the form of denture occlusion where the maxillary lingual cusps articulate with the mandibular occlusal surfaces in centric working and nonworking mandibular positions. Lingualized occlusion should not be confused with placement of the mandibular teeth lingual to the ridge crest. ${ }^{4,5}$ However, a rare situation, where combination of flabby ridge, flat ridge, and prognathic jaw relation with cross-bite situation, presents in fully edentulous patient. Therefore, modification of lingualized occlusion and without vertical overlap was needed to promote retentive, stable, and supportive complete dentures.

\section{CASE REPORT}

A 66-year-old woman came with the chief complaint of difficulty on chewing food and ill-fitting of her 

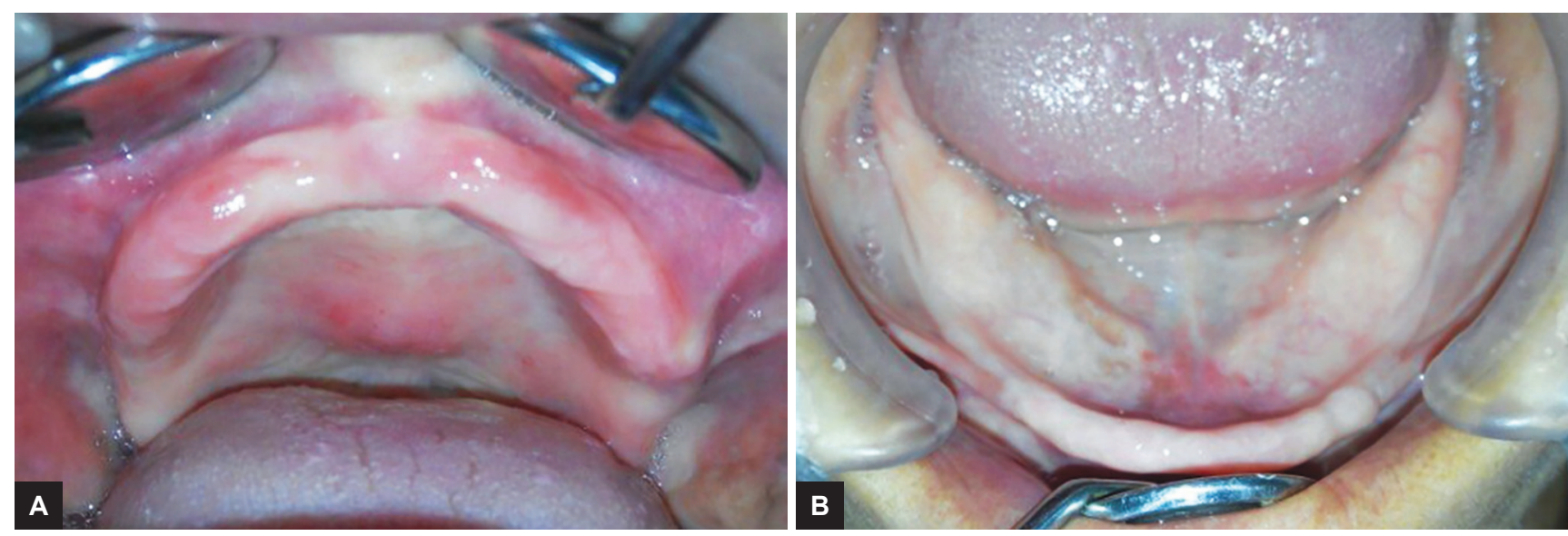

Figs 1A and B: Intraoral condition: (A) Maxilla; and (B) mandible

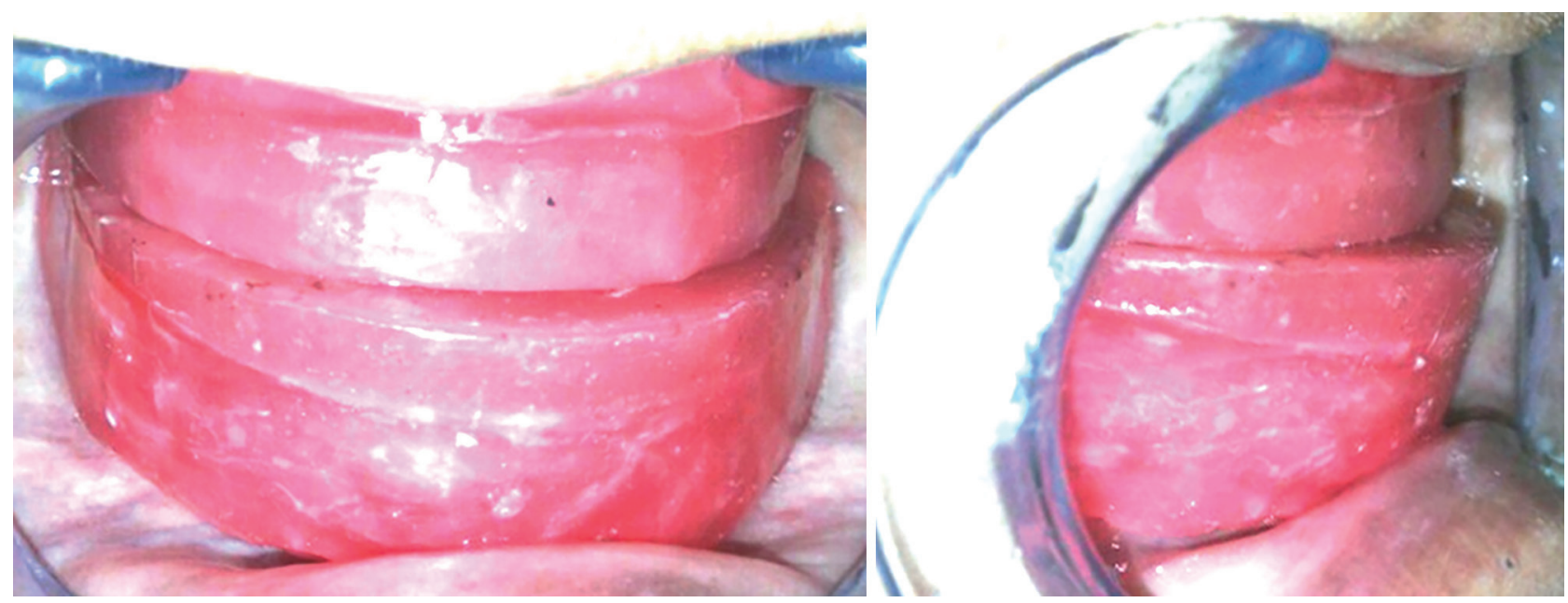

Fig. 2: Tentative VD procedure was managed to discover prognathic jaw relation

maxillary denture. She had her mandibular denture broken for around a year ago, and she had not been wearing her mandibular denture ever since. On intraoral examination, a full edentulous maxillary with flabby ridge existing on from canine to canine region was observed by palpating it with burnisher (Fig. 1A) with a flat ridge on posterior region. A full edentulous with moderate resorption on the posterior region was found in the mandible (Fig. 1B).

Preliminary impressions were taken using irreversible hydrocolloids with fabricated trays, and diagnostic casts were then obtained. A tentative VD was measured using wax bases and bite rims on diagnostic casts. The patient was led to move the mandible in physiological rest position. This method can determine jaw relation, where in this case, prognathic jaw relation with approximately $\pm 4 \mathrm{~mm}$ of horizontal overlap between maxilla and mandible, and also posterior cross-bite was discovered (Fig. 2). After that, bite rims were fixated intraorally and transferred on occludator for diagnostic purposes.

Thereafter, custom tray for final impression on maxilla was made using modified clear self-curing acrylic resin, where one sheet of wax spacer was applied on the posterior region and three sheets of wax spacer were applied on the anterior region for flabby ridge. Stoppers $(2 \times 4 \mathrm{~mm})$ were placed on the posterior region (second premolar and molar region). Stopper was not made on the anterior region to avoid the pressure on flabby ridge during impression and finger rests were placed on posterior region to provide balance pressure during impression. The tray's handle was also placed on the palatal region. The custom tray was then trimmed and polished.

Border molding on maxilla was done using green stick compound. Escape holes were made on buccal and palatal of posterior custom tray. Wax spacer on posterior was released. The final impression on posterior was taken in modified functional-compressed situation using regular body polyvinylsiloxane (PVS) (Fig. 3A). The wax spacer on anterior was then released and this custom tray allows the detection of tissue blanching on flabby region as if there is excessive pressure during custom tray try-in (Fig. 3B). This custom tray also prevents the flabby tissue being distorted during final impression. Afterward, escape holes were made close to each other using round bur 

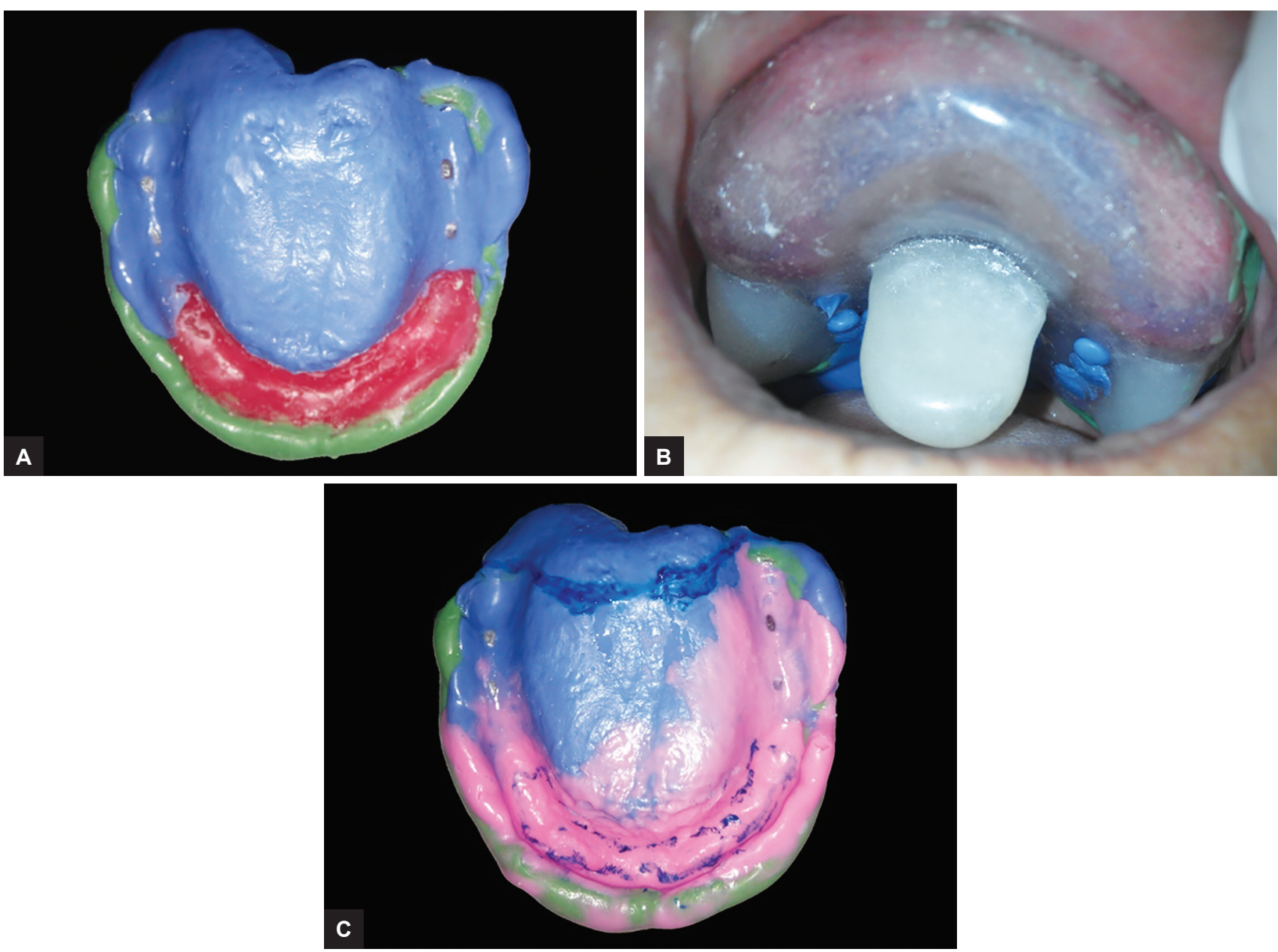

Figs 3A to C: Impression procedures on maxilla: (A) Mucofunctional impression on posterior region; (B) tissue blanching test on anterior by clear custom impression tray; and (C) mucostatic impression on anterior region

(Ø10 mm) on labial and palatal region of custom tray, and then, final impression on anterior was taken in a mucostatic situation using light body PVS. This impression procedure is known as controlled minimally displacive impression technique. Vibrating lines, hamular notches, and flabby region were marked using indelible marker. Those markings were then transferred on impression by placing back the custom tray intraorally (Fig. 3C).

Meanwhile, custom impression for final impression on mandible was made using self-curing acrylic resin, where only one sheet of wax spacer was applied on mandibular diagnostic cast. A thicker wax spacer was applied on the lingual slopes under the mylohyoid ridge to provide more space for mylohyoid muscles movement during final impression (Fig. 4A). Stoppers were placed on both sides of canine and first molar region (Fig. 4B). Finger rests were placed on posterior region, while the tray's handle was placed on the anterior region with $20 \mathrm{~mm}$ heights in vertical position and parallel with lips. After that, the custom mandibular impression tray was trimmed and polished. Border molding on mandible was done using green stick compound. Escape hole was not made on this custom tray. Then, final impression was taken using light body PVS. Afterward, evaluation of final impression's result was done, where at least three to four stoppers must be seen on the final impression's result (Fig. 4C). This impression procedure is known as controlled pressure impression technique. Then, beading on custom trays and boxing procedures were done. Dental stone was mixed and poured into the boxed impression to obtain working casts. Maxilla working cast was relieved on the flabby region and palatine suture using two sheets of customized tin foils, and fabrication of posterior palatal seal was done on it. Meanwhile, severe undercut was found on the anterior region of residual mandibular ridge and was confirmed under surveyor device.

Maxillary denture base was fabricated from heatpolymerized acrylic resin, while mandibular denture base was made from metal framework with a combination of heat-polymerized acrylic resin and soft acrylic resin, which is done only on anterior ridge with severe undercut. Occlusal bite rims were established and adjusted with neutral zone. Definitive VD was obtained. Polyvinylsiloxane material was used for VD fixation. It was then transferred in articulator for artificial teeth arrangement. 

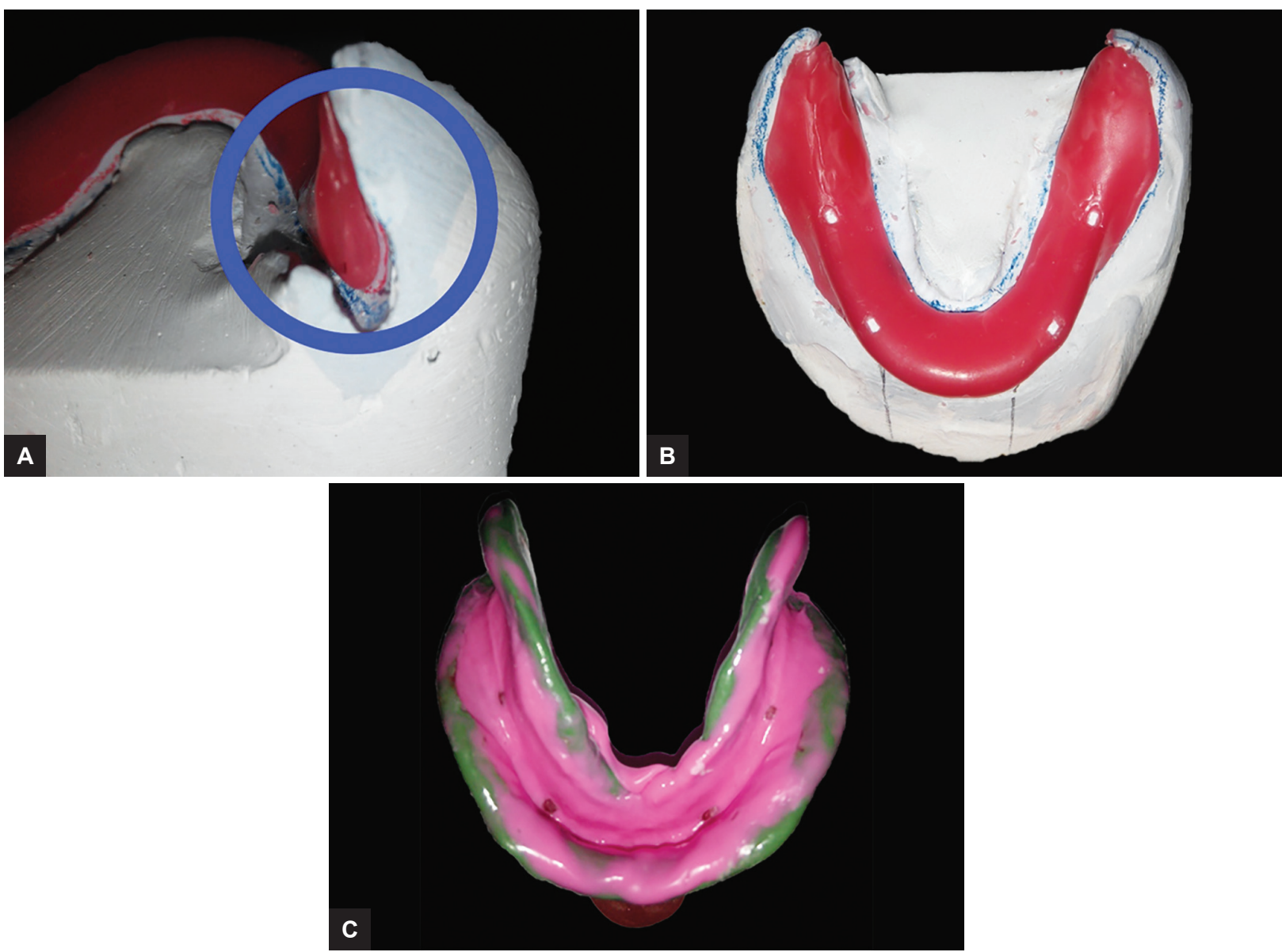

Figs 4A to C: Custom impression tray on mandible. (A) Thicker wax spacer on lingual slopes; (B) stoppers on canine and first molar region; and (C) light body PVS impression on mandible
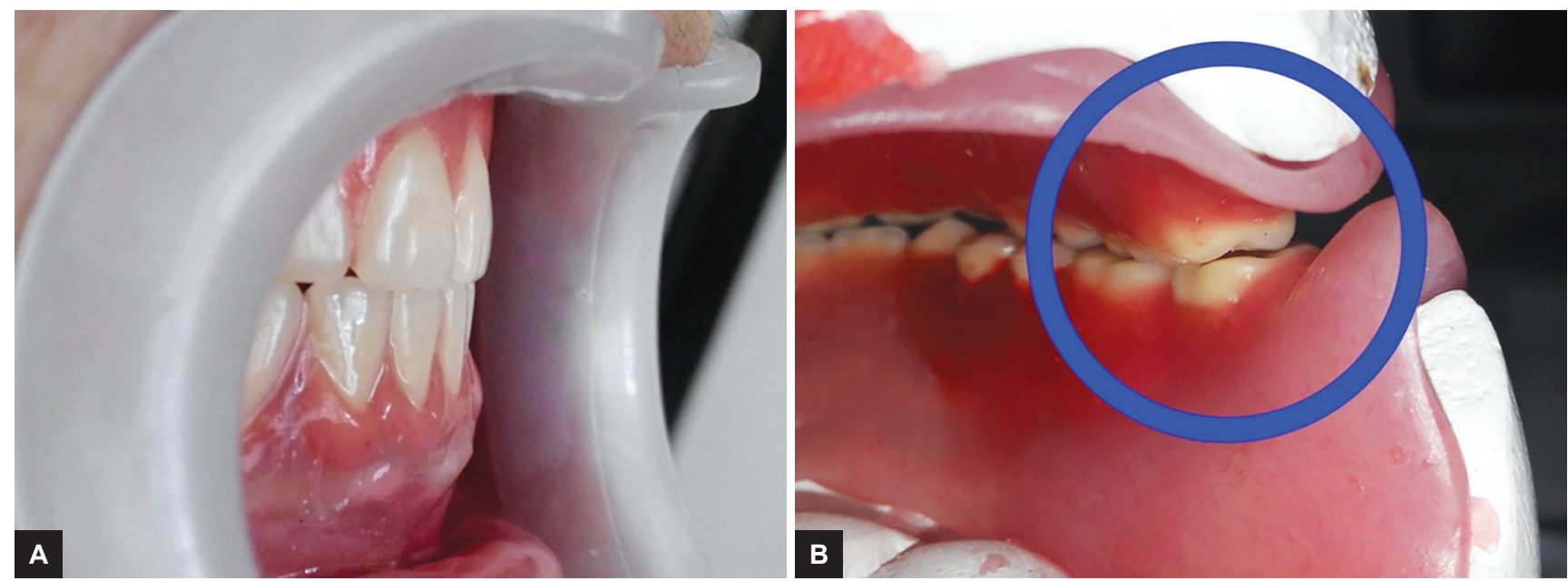

Figs 5A and B: Artificial teeth arrangement. (A) Anterior arrangement in an attempt to neutrognathic profile without vertical overlap; and $(B)$ modification of lingualized occlusion on molar region

Anterior maxillary artificial teeth were arranged slightly more to labial, while anterior mandibular artificial teeth were arranged slightly more to lingual without occlusal contact. This aims to achieve neutrognathic jaw relation and avoid pressure on flabby ridge during mastication (Fig. 5A). Lingualized occlusal scheme was used for posterior artificial teeth arrangement, where anatomic artificial teeth were used for maxilla and nonanatomic artificial teeth for mandible. However, there was posterior cross-bite situation, especially on molar region. Hence, modification of lingualized occlusion was used. In this modification, maxillary molar buccal cusps were adjusted to contact on the mandibular occlusal surface, while palatal cusps were not (Fig. 5B). 


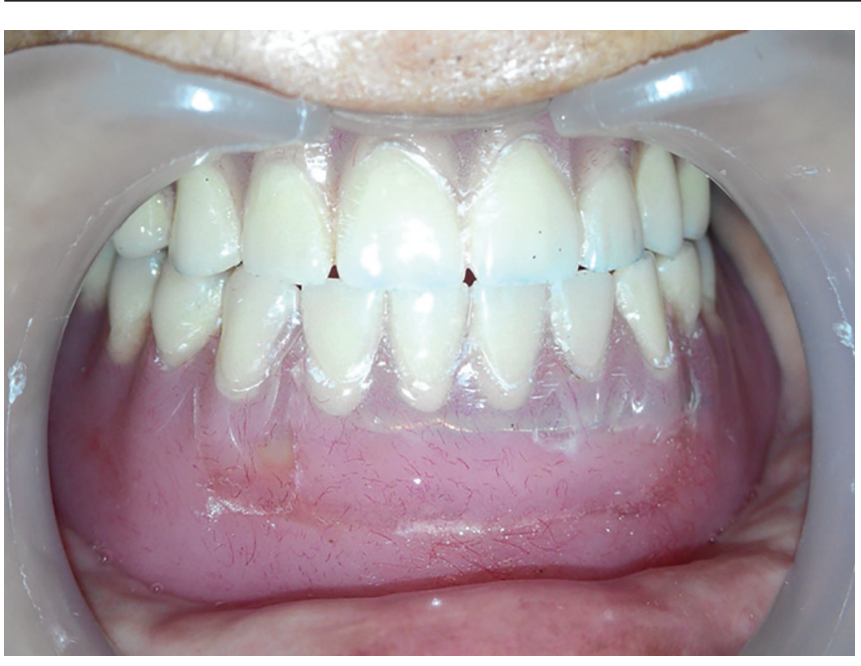

Fig. 6: Initial placement of complete dentures

Esthetic try-in and verification procedure were done. Afterward, they were delivered to laboratory for fabricating complete dentures. Remounting procedures were performed after complete dentures were fabricated in laboratory and before initial placement of complete dentures in clinic. During the initial placement, complete dentures were being evaluated, including its border extensions, border seal, retention, and esthetic values (Fig. 6). The patient was then being educated regarding the wearing, care of dentures, and cleansing procedure. Afterward, frequent recalls were done to reevaluate the complete dentures and she was quite satisfied with the retention, stability, and esthetics of the new set of complete dentures.

\section{DISCUSSION}

Surgical removal of flabby tissue is contraindicated where residual ridge support is meager. It also often results in a greater prosthodontic challenge. ${ }^{6}$ Implant-retained prostheses may offer a solution to the problems of stability and retention in fibrous ridge cases. However, they are not without their disadvantages, i.e., surgery, treatment time, and cost. A conventional prosthodontic solution may avoid these problems associated with surgery. ${ }^{7}$ Ridge augmentation by grafting is an invasive treatment option, as it carries with it the risk of resorption or rejection of graft material along with the need for additional surgery for graft harvesting. The disadvantages with this concept are anaphylactic reactions, patient discomfort, loss of firmness in some cases, and technique sensitivity. The factors involved in deciding the suitability of a patient for these surgical treatment options are age, general health, dental history, motivation, and personality. ${ }^{8}$

Prosthodontic management of a patient with a flabby maxillary ridge can be a challenging problem, and taking care to consider the influence of both the impression surface and occlusal surface in detail is paramount.
Standard mucocompressive impression techniques are likely to result in a nonretentive and unstable denture as the denture constructed on a model of the flabby tissue in a distorted state. The use of selective pressure or minimally displacive impression techniques should help to overcome some of this limitations. ${ }^{9}$ In this case report, a combination of anterior flabby and posterior flat ridge on maxilla was overcome by the custom tray for final impression where mucofunctional impression on posterior flat ridge and mucostatic impression on anterior flabby ridge was achieved to prevent tissues distortion. The clear selfpolymerized acrylic resin tray also provides the detection of flabby tissues during blanching test. Meanwhile, on other studies, clear heat-polymerized acrylic resin was used to make the final impression tray, yet it takes a lot of time to grind the intaglio surface and to try-in on the patient mouth for several times to make sure that there is no excessive pressure which will cause the flabby tissue blanched. Moreover, there was a severe undercut on anterior mandibular residual ridges, so it needs to be relieved or blocked out. Yet, it is contradictive in achieving adequate retention on mandibular residual ridges. Therefore, the use of soft heat-polymerized acrylic resin on the severe undercut region could be an advantage in providing better retention without worrying patient's discomfort of mandibular denture during function. However, it is hard to control the outspread of soft heat-polymerized acrylic resin during fabrication of denture base, which leads the metal framework to become slightly visible.

The physiologic concepts of jaw relations and occlusion are based on studies of dental and oral physiology. In clinical practice, the VD is established by first determining the length of the maxillary teeth on the occlusion rim and then making a tentative VD determination using the free-way space technique or any of the usual methods, and mounting the casts on an articulator. ${ }^{10}$ This tentative VD procedure will help practitioners to determine the diagnosis of maxillomandibular relationship. Occlusion also has a considerable influence on the outcome of every prosthodontic treatment as occlusal prematurity has destructive and destabilizing influences on dentures. Unless denture bases are adequately and evenly stabilized, it is virtually impossible to properly equilibrate the occlusion. To overcome this problem, the relative simplicity involved in the development and lingualized occlusion has gained favor among practitioners. ${ }^{11}$

Lingualized occlusion is an attempt to maintain the esthetics with advantages of the anatomic form while maintaining the mechanical freedom of nonanatomic form. Lingualized occlusion utilizes anatomic teeth for the maxillary denture and modified nonanatomic or semi-anatomic teeth for mandibular denture. To guarantee a balanced occlusion, a compensation curve is 
arranged during the setup of the mandibular posterior teeth. No posterior teeth are placed in the ascending part of the mandible to prevent protrusive dislocation of the mandibular denture. During protrusive balance, anterior teeth should be out of contact. If anterior interference occurs, either move or grind the opposing mandibular anterior teeth slightly or increase the distal incline on the mandibular molars by increasing the compensating curve. $^{12}$

After an objective comparison, the randomized prospective study by Matsumaru found that lingualized bilaterally balance occlusion was more efficient in terms of mastication and preservation of intercuspal position for patients with severe alveolar bone resorption. ${ }^{13}$ In this case, there was also cross-bite situation on the posterior region. The lingualized concept was modified in a "buccalized" one, where the maxillary buccal cusps are now adjusted to contact the mandibular occlusal surfaces, and the maxillary palatal cusps are ground so as not to take part in the articulation. Hence, modification of lingualized occlusion and without vertical overlap was conducted to overcome these complex situations, which were the combination of flabby ridge on anterior region of maxilla, flat ridge with severe undercut on anterior region of mandible, and prognathic jaw relation with posterior cross-bite. Appropriate impression and occlusal scheme are mandatory for prosthetic service in compromised conditions. However, both may be limited to clinician's decision based on knowledge and experience.

\section{CONCLUSION}

Prosthodontic rehabilitation on complex situations, such as combination of flabby ridge, flat ridge, and prognathic jaw relation with posterior cross-bite in a fully edentulous patient needs to be concerned. Modification on impression procedure, such as modified custom tray and impression technique is necessary to avoid distortion and injury on the residual ridges, and therefore, the optimal support can be obtained. The modification of lingualized occlusion without vertical overlap is also recommended for cases where flabby ridge, flat ridge, and prognathic jaw relation with posterior cross-bite existed, provided the principles of balanced articulation are strictly adhered to.

\section{CLINICAL SIGNIFICANCE}

Clear custom tray with controlled minimally displacive impression technique not only can record flabby ridge and flat ridge in their differences of compressibility but also provide visual accessibility during final impression. Modification of lingualized occlusion on posterior and without vertical overlap on anterior is recommended for a case where flabby ridge, flat ridge, and prognathic jaw relation with posterior cross-bite existed at once in complete edentulous patient.

\section{REFERENCES}

1. Shum MH, Pow EH. Management of excessive movable tissue: a modified impression technique. J Prosthet Dent 2014 Aug;112(2):387-389.

2. Yadav B, Jayna M, Yadav H, Suri S, Phogat S, Madan R. Comparison of different final impression techniques for management of resorbed mandibular ridge: a case report. Case Rep Dent 2014 Aug;2014:1-6.

3. Samyukti, Abirami G. Residual ridge resorption in complete denture wearers. J Pharm Sci Res 2016 Jun;8(6):565-569.

4. Krishna Prasad D, Rajendra Prasad B, Bardia A, Anupama Prasad D. Enhancing stability: a review of various occlusal schemes in complete denture prosthesis. Nitte Univ J Health Sci 2013 Jun;3(2):105-112.

5. Hornischer TG. The importance of lingualized occlusion. J Dent Technol 2009 Nov-Dec;26(9):20-24.

6. Dubey P, Gupta S, Pankaj, Bhatnagar A. Stock tray modification for two-stage impression technique for recording flabby ridges: report of a case series. Int J Prev Clin Dent Res 2014 Jan-Mar;2(1):75-79.

7. Crawford RW, Walmsley AD. A review of prosthodontic management of fibrous ridges. Br Dent J 2005 Dec;199(11):715-719.

8. Bindhoo YA, Thirumurthy VR, Kurien A. Complete mucostatic impression: a new attempt. J Prosthodont 2012 Apr;21(3): 209-214.

9. Jayaprakash MB, Sahu K, Khan M, Khoriya S, Jadhav S, Kendre B, Lukaram A. Management of flabby ridge cases: a challenge in clinical practice. Int J Adv Health Sci 2014 Sep;1(5):32-37.

10. Shanahan TE. Physiologic jaw relations and occlusion of complete dentures. J Prosthet Dent 2004 Mar;9(3):203-205.

11. Shah V, Dhaded S, Sajjan C. Lingualized occlusion: a better way for enhancing function and esthetic. Indian J Basic Appl Med Res 2015 Dec;5(1):484-488.

12. Kamath R, Sarandha DL, Thomas S, Sachdeva D. Lingualized occlusion: an emerging treatment paradigm for complete denture therapy: a review article. J Med Dent Sci Res 2015 Mar;2(3):6-9.

13. Abduo J. Occlusal schemes for complete dentures: a systematic review. Int J Prosthodont 2013 Jan-Feb;26(1):26-33. 